\title{
The Effect of a Vibration Absorber on the Damping Properties of Alpine Skis ${ }^{\dagger}$
}

\author{
Stefan Schwanitz 1,*, Wolfgang Griessl 2, Carlo Leilich ${ }^{2}$, Rico Krebs ${ }^{2}$, Benjamin Winkler ${ }^{2}$ and \\ Stephan Odenwald ${ }^{1}$ \\ 1 Department of Sports Equipment \& Technology, Chemnitz University of Technology, 09107 Chemnitz, \\ Germany; stephan.odenwald@mb.tu-chemnitz.de \\ 2 School of Mechanical Engineering, Chemnitz University of Technology, 09107 Chemnitz, Germany; \\ wolfgang.griessl@googlemail.com (W.G.); carlo.leilich@s2012.tu-chemnitz.de (C.L.); \\ rico.krebs@s2011.tu-chemnitz.de (R.K.); benjamin.winkler@s2013.tu-chemnitz.de (B.W.) \\ * Correspondence: stefan.schwanitz@mb.tu-chemnitz.de; Tel.: +49-371-531-36657 \\ + Presented at the 12th Conference of the International Sports Engineering Association, Brisbane, \\ Queensland, Australia, 26-29 March 2018.
}

Published: 12 February 2018

\begin{abstract}
Coupled bending-torsion vibrations at the shovel are a severe problem when running an alpine ski at high velocities on hard or icy slopes. Thus, a major goal for ski manufacturers is to dampen vibrations through a proper multi-material design and/or additional absorbers. The aim of this study was to examine the effectiveness of a particular vibration absorber on a commercial slalom ski through a series of laboratory tests as well as a subjective field evaluation. Therefore, two identical pairs of ski were used and the absorber was deactivated on one pair. Laboratory tests revealed reductions of $5 \%$ to $49 \%$ of bending vibrations on skis with activated absorber. Subjective evaluation by 6 subjects suggested minor differences in the mean of the evaluated criteria turnablity, edge grip, steering behavior and stability towards a better performance of the skis with activated absorber. Subjects were able to identify the absorber mode with a success rate of $61.1 \%$.
\end{abstract}

Keywords: skiing; bending; torsional; excitation; dynamic response; ISO 5092; ISO 6267; ISO 8783

\section{Introduction}

Alpine skis experience random vibrations during usage due to continuous excitation from uneven topology of the sliding surface. Level and kind of excitation varies depending on several conditions such as snow and slope properties, rider velocities or steering maneuvers [1,2]. The dynamic response of the multi-material structure ski is a major quality criterion for engineers and designers [2]. It is related to the aspects of performance as well as of safety of the ride because it effects the control of the ski. Control is limited if vibrations lead to a reduced effective length of the edge in contact with the snow. By exceeding a critical velocity, continuous excitation through humps or icy patches leads to unexpected responses of the ski and thus, it will not follow the desired or anticipated curve arc [1].

High mass, high torsional and bending stiffness, long length or high damping can improve the riding stability of skis at high velocities. This can be observed in skis for competitive use in downhill or Super-G [1,2]. In contrast, recreational skiers prefer to ride short and lightweight skis with better agility but less bending stiffness [1]. Thus, the goal of engineers is to develop additional damping mechanisms or to use smart materials to achieve capable skis in a multi-target optimization $[1,3]$. Damping is the ability of a ski to reduce vibration within a given time. The aim of this study was to examine the effectiveness of a particular vibration absorber through a series of laboratory tests as well as a subjective field evaluation. 


\section{Materials and Methods}

\subsection{Test Ski}

Two pairs of the slalom ski Racetiger Speedwall SL UVO of the season 2016/17 were provided by Völkl Sports GmbH \& Co. KG (Straubing, Germany). The weight of one ski was $3200 \mathrm{~g}$ including bindings (Marker Xcell 12.0, Marker Dalbello Völkl (International) GmbH, Baar, Switzerland). The nominal length was $1650 \mathrm{~mm}$, sidecut from tip to tail 123-68-104 $\mathrm{mm}$ and radius $12.7 \mathrm{~m}$. Its intended use is $100 \%$ on-piste skiing in slalom/race style of riding and it addresses the experienced athlete. The ski model features a so-called Ultimate Vibration Object (UVO, see Figure 1). This patented technology (EP 2368607 A1) intends to damp vibrations of the shovel in bending and torsion modes by adding a counterweight (weight $53 \mathrm{~g}$ ) to the tip [4]. The counterweight is able to move freely relative to the shovel approximately $3 \mathrm{~mm}$ from center towards tip or tail as well as to left or right. To examine the efficacy of the UVO, it was locked at one pair of skis by closing the gap in between the counterweight and the plastic frame that connects the UVO-unit to the ski.

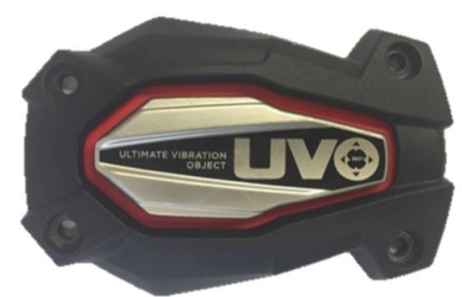

(a)

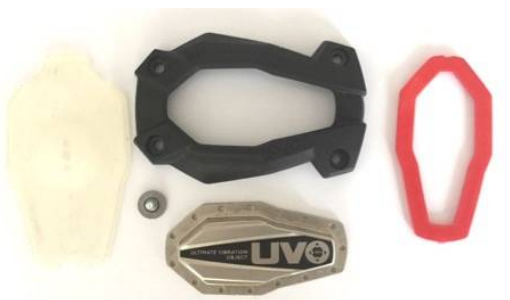

(b)

Figure 1. (a) UVO assembly, (b) unmounted, dimensions of the plastic frame (black) approx. $80 \times 50 \mathrm{~mm}$.

\subsection{Determination of Bending Stiffness}

In order to obtain the bending stiffness of the middle part of the skis cm, a modified laboratory test method according to ISO 5902:2013 [5] was applied. The skis were laid on two supports (distance nominal length $\mathrm{ln}_{\mathrm{n}}-280 \mathrm{~mm}$ ) and firmly clamped at a distance of $50 \mathrm{~mm}$ to the ski rear end. Then, a preloading of $20 \mathrm{~N}$ was applied using a manually driven actuator through a dummy boot sole, which was attached to the ski binding. Load was measured and displayed to the operator through a load cell (type 8435, Burster Präzisionsmesstechnik GmbH, Gernsbach, Germany). The point of force application was in the center between both supports. At the same point deflection of the ski at preload $\mathrm{d}_{\mathrm{F}=20 \mathrm{~N}}$ was measured using a Laser displacement sensor (type LK-G152, Keyence Deutschland GmbH, Neu-Isenburg, Germany). Then load was increased until $300 \mathrm{~N}$ and deflection $\mathrm{dF}=300 \mathrm{~N}$ was obtained. Finally, cm was calculated:

$$
c_{M}=\frac{300 N-20 N}{d_{F=300 N}-d_{F=20 N}}
$$

\subsection{Investigation of the First Natural Bending Mode}

The second test followed the guidelines provided in ISO 6267:1980 that addresses the measurement of bending vibrations of the shovel [6]. Therefore, the rear part of the ski was clamped to a support. The shovel remained not fixed. A Laser displacement sensor (type LK-G152, Keyence Deutschland $\mathrm{GmbH}$, Neu-Isenburg, Germany) was positioned perpendicular to ski base at $300 \mathrm{~mm}$ towards tip from half of $l_{\mathrm{N}}$ Free oscillations of the ski after release from $2.5 \mathrm{~mm}$ deflection were recorded. The 1st natural frequency $\mathrm{f}_{\mathrm{n}, 1}$ was retrieved from the time interval between 30 oscillations. Furthermore, half life $t_{1 / 2}$ was obtained. Half life is the time interval that is covered between the first amplitude and the amplitude that is less than $50 \%$ of the first. The measurements were repeated 10 times for each UVO condition. 


\subsection{Investigation of the Dynamic Response to Forced Oscillation}

A third laboratory test was performed in order to expose the skis to forced oscillations by a single-point force at the tip. Skis were clamped at the bindings, assuming they were remaining in constant contact with the snow surface. An electrodynamic exciter (type TMS-K2007E01 Mini Smart Shaker, The Modal Shop Inc., Sharonville, OH, USA) was placed underneath the ski shovel. It induced sinusoidal and rectangle shaped oscillations to model the rough snow surface [7]. Beginning from $1 \mathrm{~Hz}$ frequency of the stimulus was increased by increments of $1 \mathrm{HZ}$ every $10 \mathrm{~s}$ up to $30 \mathrm{~Hz}$. Amplitude of the oscillations was constant at $13 \mathrm{~mm}$ peak to peak, which was the physical maximum of the exciter.

During the tests, acceleration of the shovel was recorded (sampling rate $1000 \mathrm{~Hz}, 500 \mathrm{~Hz}$ lowpass anti-aliasing filter) using an uniaxial accelerometer (type ADXL72, Analog devices Inc., Norwood, MA, USA) that was attached closely to the UVO. Running root mean square (RMS) values of the acceleration were calculated for each time interval of constant excitation frequency and for both kinds of excitation.

\subsection{Subjective Evaluation}

For subjective evaluation, a questionnaire was developed following the guidelines in ISO 8783:2015 [8]. Instead of a ranking scale with 5 degrees for each criterion, visual analog scales (0 to $100 ; 0=$ very poor $; 100=$ very good) were used to evaluate the following characteristics:

- turnability: indicates the "ease of turning, the less force and movement required, the greater the turnability" [8],

- edge grip: "the ability of the ski to hold on hard surfaces" [8],

- steering behavior: "execution and completion of a turn to change direction" [8],

- stability: "maintaining direction at a given speed when turning" [8],

- overall: the mean value of the four criterions mentioned above.

The field-testing took place on a public slope (length $200 \mathrm{~m}$; grade black/difficult) in the Fichtelberg ski resort (Oberwiesenthal, Germany). To ensure comparable boundary conditions, testing was performed within $4 \mathrm{~h}$ of a single day. Six subjects (male, age $24 \pm 3$ years, height $184 \pm 7$ $\mathrm{cm}$, weight $74.2 \pm 8.5 \mathrm{~kg}$, boot sole length $330 \pm 12 \mathrm{~mm}$ ) volunteered in this study. All participants provided written informed consent. The study was in accordance with the Declaration of Helsinki. In a self-assessment to a four level scale, the subjects indicated themselves as advanced learner $(n=4$, level 2), expert skier ( $n=1$, level 3) or master ( $n=1$, level 4). None of them was a beginner (level 1).

The evaluation was divided in two parts. In part A, the damping system of both skis were either activated (A-A) or inactive (I-I). For each person three runs were performed. In part B, subjects obtained on one leg a ski with an active vibration damper and on the other side with an inactive (AI or I-A). Likewise, for each person three runs were performed. In part $\mathrm{A}$, the evaluation of the criterions followed for every pair of skis, in part B, for every ski separately. Furthermore, in part A, subjects were asked to indicate whether they rode a pair of skis with activated UVO or not. Arithmetic means and standard deviations of six subjects were calculated for all criterions and both study designs. Additionally, analyses of variance were performed in order to test statistically significant differences in the means between active and inactive UVO conditions.

\section{Results}

\subsection{Elastic Properties: Bending Stiffness}

Despite identical skis of the same design and the same model, there are slight differences between the individual skis with regard to bending characteristic. Within the four individual skis out of two pairs, the resistance against elastic deformation см differed in between 5.28 and $5.71 \mathrm{~N} / \mathrm{mm}$. Arithmetic mean was $5.49 \pm 0.18 \mathrm{~N} / \mathrm{mm}$. Due to low variation each ski was regarded as being of identical bending stiffness properties. 


\subsection{Dynamic Properties: 1st Bending Mode}

The arithmetic means and standard deviations of the results obtained in ISO 6267:1980 test are given in the following Table 1 . Skis with an active UVO revealed a $5 \%$ lower 1st natural frequency than the inactive UVO condition. Half life divided in approximately half. Variability by means of standard deviations between the repeated measurements was comparably low.

Table 1. Results of the tests according to ISO 6267:1980 concerning the first natural bending mode; arithmetic mean and standard deviation of 10 measurements with each UVO-condition.

\begin{tabular}{ccc}
\hline Condition & 1st Natural Frequency $\mathbf{f}_{\mathbf{n}, 1} \mathbf{( H z )}$ & Half Life $\mathbf{t}_{1 / 2}(\mathbf{s})$ \\
\hline Active UVO & $11.39 \pm 0.14$ & $0.204 \pm 0.051$ \\
Inactive UVO & $12.02 \pm 0.06$ & $0.399 \pm 0.080$ \\
\hline
\end{tabular}

\subsection{Dynamic Properties: Response to Forced Oscillations}

In order to compare the dynamic responses of the skis to forced excited vibrations the running RMS of the acceleration at the ski tip is plotted over the range of excitation frequencies that have been studied. Running RMS is lower for the active UVO in both cases until approx. $21 \mathrm{~Hz}$. In general, dynamic response of the skis was greater in case of the rectified stimulus (see Figure 2). With respect to RMS of the whole timespan, the activated UVO decreased vibrations in case of sinusoidal excitation for $17 \%$ ( $3.92 \mathrm{~g}$ to $3.25 \mathrm{~g}$ ), while rectangular signal forms lead to differences of $5 \%$ between active and inactive UVO condition (5.94 g to $5.64 \mathrm{~g}$ ).

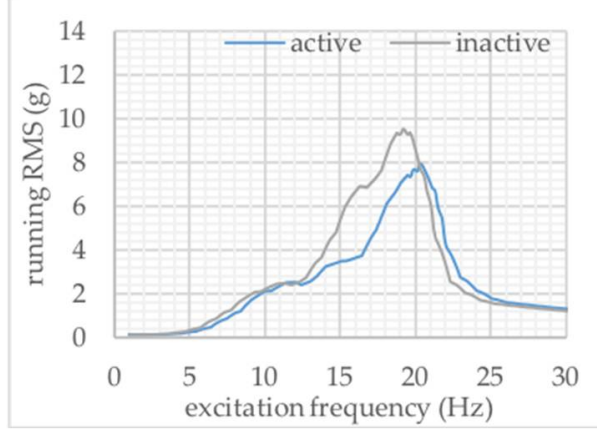

(a)

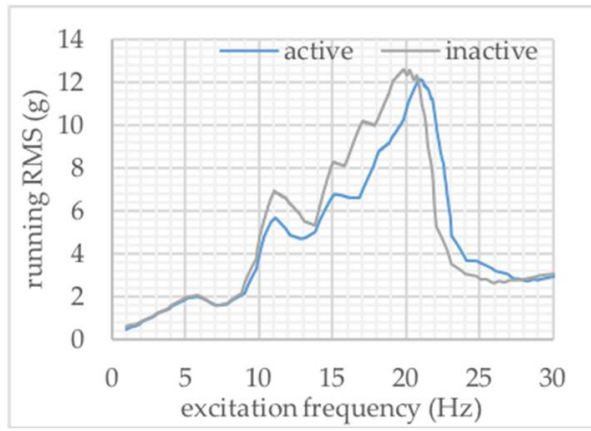

(b)

Figure 2. Dynamic response to (a) sinusoidal and (b) rectified excitation.

\subsection{Subjective Evaluation}

As a general observation, it has to be stated, that the subjective evaluation showed high variability in every parameter (see Table 2). Subjects were able to identify correctly whether the UVO was enabled or deactivated in 11 out of 18 cases. In only two cases, a subject gave an incorrect answer and in five cases, the subject was unable to decide. This gives a success rate of $61.1 \%$. Mean values of the evaluated criterions (see also Figure 3) indicate differences between both conditions, with a maximum difference of $5 \%$. Analyses of variances did not reveal statistically significant differences. Interestingly, ratings were higher for each criterion and both UVO conditions in test design A.

Table 2. Arithmetic means and standard deviations of the ratings of 6 subjects for all criterions and both study designs of the subjective evaluation (numbers in \%).

\begin{tabular}{ccccccc}
\hline UVO Condition & Test Design & Stability & Turnability & Edge Grip & $\begin{array}{c}\text { Steering } \\
\text { Behavior }\end{array}$ & Overall \\
\hline Active & A & $75 \pm 9$ & $69 \pm 13$ & $73 \pm 8$ & $72 \pm 9$ & $72 \pm 10$ \\
Inactive & A & $71 \pm 12$ & $70 \pm 14$ & $74 \pm 8$ & $70 \pm 12$ & $71 \pm 11$ \\
Active & B & $65 \pm 22$ & $69 \pm 11$ & $68 \pm 12$ & $70 \pm 10$ & $68 \pm 12$ \\
Inactive & B & $64 \pm 20$ & $67 \pm 10$ & $67 \pm 13$ & $64 \pm 11$ & $66 \pm 12$ \\
\hline
\end{tabular}




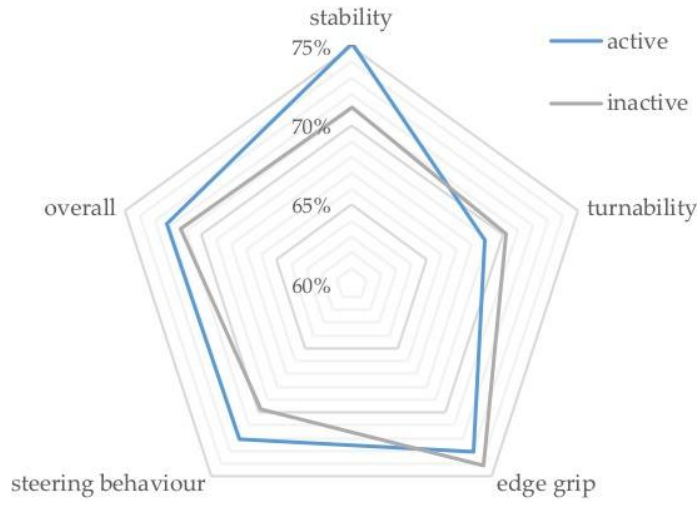

(a)

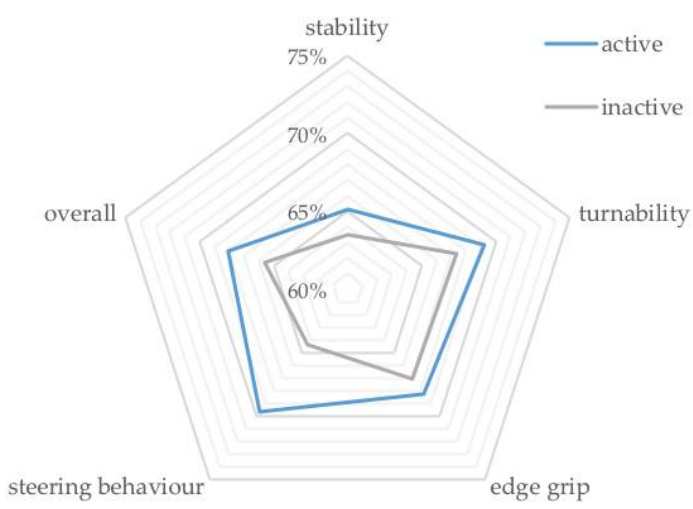

(b)

Figure 3. Evaluation of the UVO in (a) pairs of identical conditions and (b) pairs with mixed conditions.

\section{Discussion}

Wills (2014) investigated the vibration damping behavior of nine skis of six brands and several years of release from 1980 to 2014 [9]. The average half life period of $0.523 \pm 0.138 \mathrm{~s}$ was slightly higher compared to the test samples of this study with locked UVO and by far higher compared to the samples with UVO activated. The frequency of the first natural bending mode was found to be slightly below the values in literature [2,9]. The results (see Figure 4) show that ski construction is progressing in general and that active vibration damping of the first bending mode is possible by adding small and lightweight damping devices such as the UVO system.

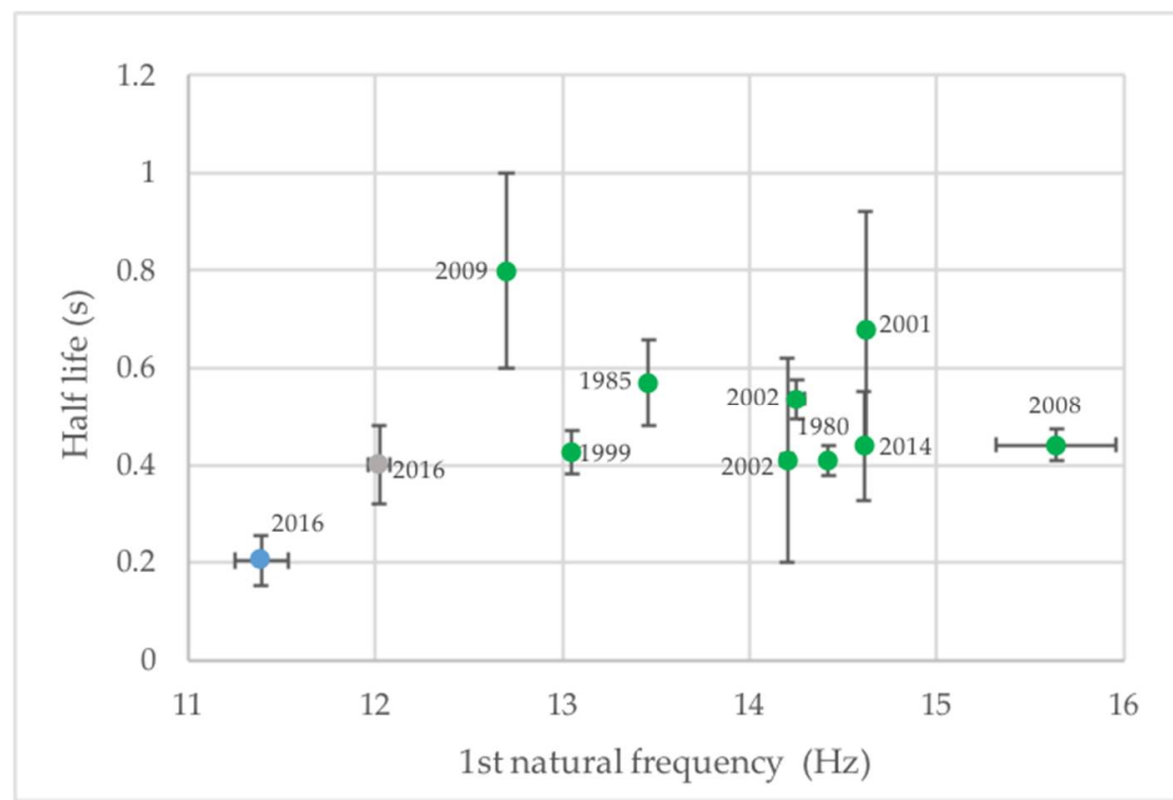

Figure 4. Comparison of test results (arithmetic means and standard deviations) concerning the 1st natural frequencies and half lifes obtained in this study (blue and grey marker) to literature (green dots) [9], year of release of the particular ski as additional information.

Dynamic responses of the skis were different depending on the form of the point force excitation. A probably more realistic rectified stimulus [2] lead to much higher RMS values than the smooth sinusoidal stimulus. On the other hand, the differences between active and inactive vibration absorber $(5 \%)$ were much smaller compared to the testing according ISO 6267:1980 (49\%) or sinusoidal excitation (17\%).

The higher absolute values for the subjectively evaluated criteria in part A compared to part $\mathrm{B}$ indicate, that riding two different skis respectively ski conditions at the same time does lead to a more 
insecure feeling of the subjects. Thus, this approach cannot be recommended for further studies due to ethical reasons. However, the results of the subjective evaluations underlie to several limitations. Test conditions were not as required by ISO 8783. Instead of hard and well-prepared snow conditions [8], slopes were soft and hilly. This affected clear ratability of differences in riding characteristics in general. Moreover, the UVO technology is intended to absorb small oscillations of the shovel on hardpacked or icy snow. Another limitation is that the perception skills of test rides were not evaluated before, which is advisable for future studies. In addition, the self-assessed skiing ability of most of the subjects was below the target group of the commercial product that was tested within this study. It is questionable if all subjects were able to perform the proper ski techniques according to the test criteria, as it is required by the standard [8]. Furthermore, none of the testers was experienced in ski product evaluation.

\section{Conclusions}

The study proved the feasibility to reduce vibrations of alpine skis by use of small, relatively lightweight external damping devices. The damping of the 1st bending vibration mode $(10$ to $15 \mathrm{~Hz})$ was clearly influenced by the activated UVO device. With increased external validity of the test design, differences between skis with active and inactive UVO extenuated. Further research has to be done on the isolated torsional vibration modes and on coupled vibrations. In addition, the setup for subjective field-testing has to be refined.

Acknowledgments: This study has been conducted within the program Master of Science in Sports Engineering at Chemnitz University of Technology. The authors would like to thank Christian Maiwald (Department of Research Methodology and Data Analyses) and his team for their contribution and support in developing the test design, performing the data processing and interpretation of the subjective evaluations. Furthermore, the authors would like to acknowledge Tobias Heil and Robert Hecht (Völkl Sports GmbH \& Co. KG, Straubing, Germany) for supporting this study with two pairs of skis and useful technical information about the UVO technology.

Conflicts of Interest: The authors declare no conflict of interest. The supporting company Völkl Sports GmbH \& Co. KG (Straubing, Germany), which provided two pairs of skis including bindings at no charge, had no role in the design of the study; in the collection, analyses, or interpretation of data; in the writing of the manuscript, and in the decision to publish the results.

\section{References}

1. Foss, G.C.; Glenne, B. Reducing On-Snow Vibrations of Skis and Snowboards. Sound Vib. 2007, 41, 22-27.

2. Fanti, G.; Basso, R.; Montaui, V. Damping Measurement of Bending Vibration in Alpine Skis: An Improvement of Standard ISO 6267. Appl. Mech. Mater. 2006, 5-6, 199-206, doi:10.4028/www.scientific.net/AMM.5-6.199.

3. Nash, B. Engineering a Smooth Ride: Creating the Perfect Ski through Shaping and Vibration Damping. Illumin Rev. Eng. Everyday Life 2003, 4, 1-5.

4. Ametsbichler, A.; Gleissner, T.; Heil, T. Snow Glide Board, in Particular a Ski, with Binding Rails Associated with a Dampening System. EP 2368607 A1, 28 September 2011.

5. ISO (International Organization for Standardization). ISO 5902:2013 Alpine Skis-Determination of Elastic properties; ISO: Geneva, Switzerland, 2013.

6. ISO (International Organization for Standardization). ISO 6267:1980 Alpine Skis-Measurement of Bending Vibrations; ISO: Geneva, Switzerland, 1980.

7. Chapman, R.; Hegewald, KA; Humphreys, S.; Meckstroth, S. Vibration analysis of an alpine ski. J. Acoust. Soc. Am. 1993, 94, 1856, doi:10.1121/1.407669.

8. ISO (International Organization for Standardization). ISO 8783:2015 Alpine Skis-Guidelines for Conducting Slope Performance Tests; ISO: Geneva, Switzerland, 2015.

9. Wills, T. Alpine Ski Vibrations Analysis; Project Report; Sheffield Hallam University: Sheffield, UK, 2014.

(C) 2018 by the authors. Licensee MDPI, Basel, Switzerland. This article is an open access article distributed under the terms and conditions of the Creative Commons Attribution (CC BY) license (http://creativecommons.org/licenses/by/4.0/). 